\title{
TESTES DE CONFIABILIDADE DOS MÉTODOS DE DATAÇÃO POR TERMOLUMINESCÊNCIA (TL) E LUMINESCÊNCIA OTICAMENTE ESTIMULADA (OSL)
}

\author{
Shigueo Watanabe* \\ Sônia Hatsue Tatumi** \\ Thiago Michel de Brito Farias* \\ Susana de Oliveira Souza *** \\ Roseli Fernandes Gennari* \\ Ruth Kunzl **** \\ Deisi Sunderlick Eloy de Farias*****
}

WATANABE, S.; TATUMI, S.H.; FARIAS, T.M.B.; SOUZA, S.O.; GENNARI, R.F.; KUNZL, R.; FARIAS, D.S.E. Testes de confiabilidade dos métodos de datação por termoluminescência (TL) e luminescência oticamente estimulada (OSL). Rev. do Museu de Arqueologia e Etnologia, São Paulo, 15: 383-391, 2005.

RESUMO: Para os pesquisadores de arqueologia e geologia, dentre outras ciências, a informação sobre a idade dos materiais em estudo é importante. Há várias técnicas de datação, a começar do muito conhecido método de radiocarbono, apropriado para amostras que absorveram carbono no passado, outros se baseiam no decaimento radioativo de diferentes radioisótopos com meia vida apropriada, e aqueles que se baseiam na deposição parcial de energia de uma radiação ionizante, incidente sobre o material cuja idade se quer determinar. Fazem parte da última classe de métodos o de ressonância paramagnética eletrônica (EPR), de termoluminescência (TL) e o de luminescência oticamente estimulada (LOE ou OSL-optically stimulated luminescence), que são muitos usados pela sua facilidade de aplicação e alcance (Ka a Ma). Contudo, às vezes surgem resultados que têm causado dúvidas quanto à confiabilidade da técnica TL e OSL.

O objetivo deste trabalho consiste em demonstrar que as medidas feitas em dois dos laboratórios mais ativos em datação do país, da dose acumulada em três peças cerâmicas, pela técnica de termoluminescência, apresentaram resultados coerentes, comprovando a confiabilidade da técnica. Este teste pode ser estendido às técnicas de OSL e EPR e será objeto de um trabalho futuro.

UNITERMOS: Termoluminescência - Datação-OSL - Cerâmica.

(*) Instituto de Física, Universidade de São Paulo, SP, Brasil. Shigueo Watanabe watanabe@if.usp.br ; Roseli Fernandes Gennari rgennari@dfn.if.usp.br; Thiago Michel de Brito Farias tfarias@dfn.if.usp.br (**) Faculdade de Tecnologia, FATEC, SP, Brasil. tatumi@fatecsp.br

\footnotetext{
(***) Universidade Federal de Sergipe - UFSE, Brasil. sosouza@fisica.ufs.br (****) UNESP- Universidade Estadual Paulista,Presidente Prudente - SP. ruth@prudente.unesp.br $(* * * * *)$ Universidade Sul de Santa Catarina (UNISUL) - SC. deisisf@unisul.br
} 


\section{Introdução}

(Aitken 1995; Cameron et al. 1968; Ikeya 1993; Mc Keever 1988; Chen, Mc Keever 1997; Krbetschek et al. 1997; Roque et al. 2004).

A termoluminescência (TL), ou luminescência termicamente estimulada, é um fenômeno observado em cristais iônicos (como o quartzo e a calcita) e em alguns vidros, e consiste na emissão de luz, em geral na região do visível, por um material aquecido, tendo sido este previamente irradiado com radiação ionizante. Na LOE (ou OSL optically stimulated luminescence), a emissão da luz é estimulada pela luz ultravioleta.

Tanto na TL como na OSL é fundamental que o material tenha sido irradiado antes da emissão da luz. Essa luz é, então, convertida em corrente elétrica, em uma válvula fotomultiplicadora e é representada sob a forma de uma curva, na qual no eixo vertical é colocada a intensidade da luz e no eixo horizontal, a temperatura de aquecimento. A curva resultante é chamada de curva de emissão, caracterizada por apresentar, na maioria dos casos, dois ou mais picos. O máximo de um pico se dá numa temperatura $\left(T_{p}\right)$, um parâmetro importante, pois, um pico $\mathrm{T}_{\mathrm{p}}$ inferior a $150^{\circ} \mathrm{C}$ é instável em temperatura ambiente e não pode ser usado nas datações. No caso de quartzo, o pico que ocorre em $325^{\circ} \mathrm{C}$ (ou $375^{\circ}$ quando este está presente) é o normalmente usado.

A intensidade da luz TL (ou OSL) emitida depende da dose da radiação, esse fato constitui a base da datação.

No caso de objetos cerâmicos arqueológicos, sabe-se que seu preparo envolve o aquecimento, em temperatura acima de $500^{\circ} \mathrm{C}$ a $600^{\circ} \mathrm{C}$, de um molde de argila. Essa queima elimina totalmente a TL anteriormente existente na argila e estabelece o zero do relógio arqueológico. Em geral, um vaso cerâmico arqueológico é encontrado no solo, a uma profundidade de 10 a mais de $100 \mathrm{~cm}$. A partir do momento em que ficou soterrado, começa a receber a radiação (gama, beta e alfa) proveniente de desintegração de elementos radioativos pertencentes a uma série que começa $\mathrm{com}^{238} \mathrm{U}$ e de outra encabeçada pelo ${ }^{232} \mathrm{Th}$.

A idade da cerâmica refere-se, portanto, ao período de tempo iniciado na queima do molde de argila até a coleta da cerâmica do sitio arqueológico.

Durante o tempo em que fica no subsolo a cerâmica é irradiada, como foi previamente mencionado, e se induz a TL (ou OSL). O valor desta luz TL induzida, dividido pela luz TL induzida por unidade de dose (1Gy) fornece a chamada dose acumulada $\left(\mathrm{D}_{\mathrm{ac}}\right)$ (ou dose equivalente - $\mathrm{D}_{\mathrm{eq}}$ ).

Na datação, um outro dado importante é a taxa anual de dose da radiação natural, ou simplesmente dose anual $\left(D_{a n}\right)$. A taxa de dose anual do local de coletada da amostra arqueológica ou geológica pode ser obtida ou a partir das concentrações de urânio, tório e potássio do solo e da própria cerâmica, ou a partir da determinação da TL acumulada em dosímetros TL enterrados no local de estudo por dois a seis meses.

No presente trabalho as concentrações de urânio e tório nas cerâmicas investigadas foram determinadas pelo método de ICP-MS (Espectrometria de Massas com Plasma).

O U-238 e o Th-232 são os elementos radioativos originários das séries radioativas de urânio e de tório, respectivamente. As energias das partículas alfa, beta e gama emitidas em cada série são conhecidas, assim a dose de radiação por ano pode ser calculada através da determinação dos teores de U-238 e Th-232.

O K-40 é igualmente radioativo (emissor â e ã).

TABELA 1

Concentrações de U-238, Th-232 e de $\mathrm{K}_{2} \mathrm{O}$ nas cerâmicas estudadas

\begin{tabular}{cccc}
\hline \hline $\begin{array}{c}\text { Elemento } \\
\text { Radioativo }\end{array}$ & CE-PP & QB4N3L3 & QE3N3L3 \\
\hline${ }^{238} \mathrm{U}(\mu \mathrm{g} / \mathrm{g})$ & $8,3 \pm 1,4$ & $4,2 \pm 1,2$ & $12,7 \pm 2,9$ \\
${ }^{232} \mathrm{Th}(\mu \mathrm{g} / \mathrm{g})$ & $12,9 \pm 1,3$ & $4,31 \pm 0,09$ & $5,8 \pm 0,7$ \\
$\mathrm{~K}_{2} \mathrm{O}(\%)$ & $1,4 \pm 0,4$ & $1,3 \pm 0,5$ & 1,5 \\
$\mathrm{D}_{\text {an }}(\mathrm{mGy} / \mathrm{ano})$ & 1,91 & 1,62 & 2,51 \\
\hline
\end{tabular}

Conhecendo a porcentagem de $\mathrm{K}_{2} \mathrm{O}$, determina-se a contribuição desse elemento à dose anual. Tanto as doses anuais de U-238 e Th-232 como de K-40, podem ser computadas conforme Ikeya (1993).

A Tabela I abaixo apresenta as concentrações de U, Th e K e a taxa de dose anual.

\section{Objetivo deste trabalho e materiais}

Alguns arqueólogos brasileiros têm duvidado da validade dos métodos TL e OSL de datações arqueológicas. A fim de dirimir esta dúvida, no presente trabalho foi feita uma comparação de datação realizada pelos laboratórios (Tatumi et al. 1998; Sastry et al. 2004), que mais se têm engajados 


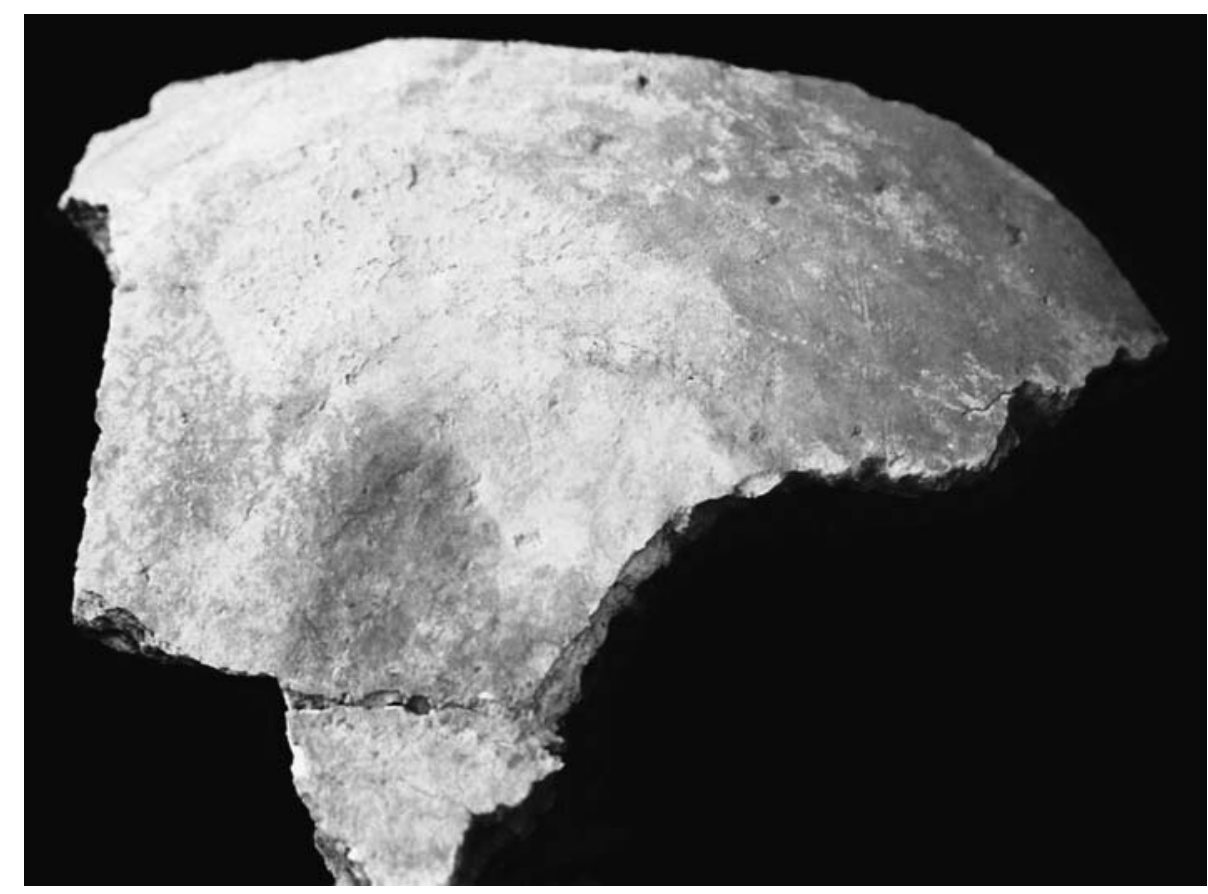

Fig. 1 - Cerâmica de Presidente Prudente.

nessa tarefa, a saber, da Faculdade de Tecnologia, da UNESP e do Instituto de Física da USP.

A Prof ${ }^{a} \operatorname{Dr}^{a}$ Ruth Kunzli, da Faculdade de Ciências Tecnológicas da UNESP-Presidente Prudente, forneceu, para o presente trabalho, uma peça de cerâmica, encontrada num sítio arqueológico na vizinhança de Presidente Prudente e medindo cerca de $20 \mathrm{~cm}$ x $25 \mathrm{~cm}$ (Fig. 1).

A Prof ${ }^{a} \operatorname{Dr}^{\mathrm{a}}$ Deisi S. E. Farias, da Universidade Sul de Santa Catarina, forneceu peças cerâmicas, coletadas num sítio arqueológico na região de Tubarão, Santa Catarina, identificadas por QE3N3L3, QE4B4L3. (Fig. 2).

A cerâmica de Presidente Prudente, aqui denominada de CE-PP, foi dividida em quatro partes. Uma para medidas na FATEC, uma para o laboratório de datação da Universidade Federal de Sergipe, a terceira para medidas de EPR, na Faculdade de Filosofia Ciências e Letras da USP-Ribeirão Preto e, a quarta para o LACIFID (Laboratório de Cristais Iônicos, Filmes Finos e Datação) do Instituto de Física da USP.

O solo brasileiro é rico em ferro e as cerâmicas contêm uma quantidade bastante considerável de ferro na forma de magnetita $\left(\mathrm{Fe}_{2} \mathrm{O}_{3}\right)$. Acontece que a magnetita apresenta a chamada interação spin-spin, que produz um sinal de EPR fortíssimo, encobrindo os demais sinais normalmente usados nas datações. Portanto, após a constatação desse sinal de interferência, a participação do grupo, chefiado pelo Prof. Dr. Oswaldo Baffa de Ribeirão Preto foi cancelada.

Na Universidade Federal de Sergipe, o grupo de datação coordenado pela $\operatorname{Prof}^{a} \operatorname{Dr}^{\mathrm{a}}$ Susana Oliveira de Souza estava com problemas no equipamento principal, por isso, essa participação também foi cancelada.

Ficaram, assim, as medidas para comparação a cargo dos laboratórios datação da FATEC e do IFUSP.

\section{Preparação das amostras para as medidas}

Em ambiente escuro, as amostras de cerâmicas coletadas foram lixadas, pulverizadas em almofariz de porcelana e, então, peneiradas até a obtenção de granulometria entre 0,080 e 

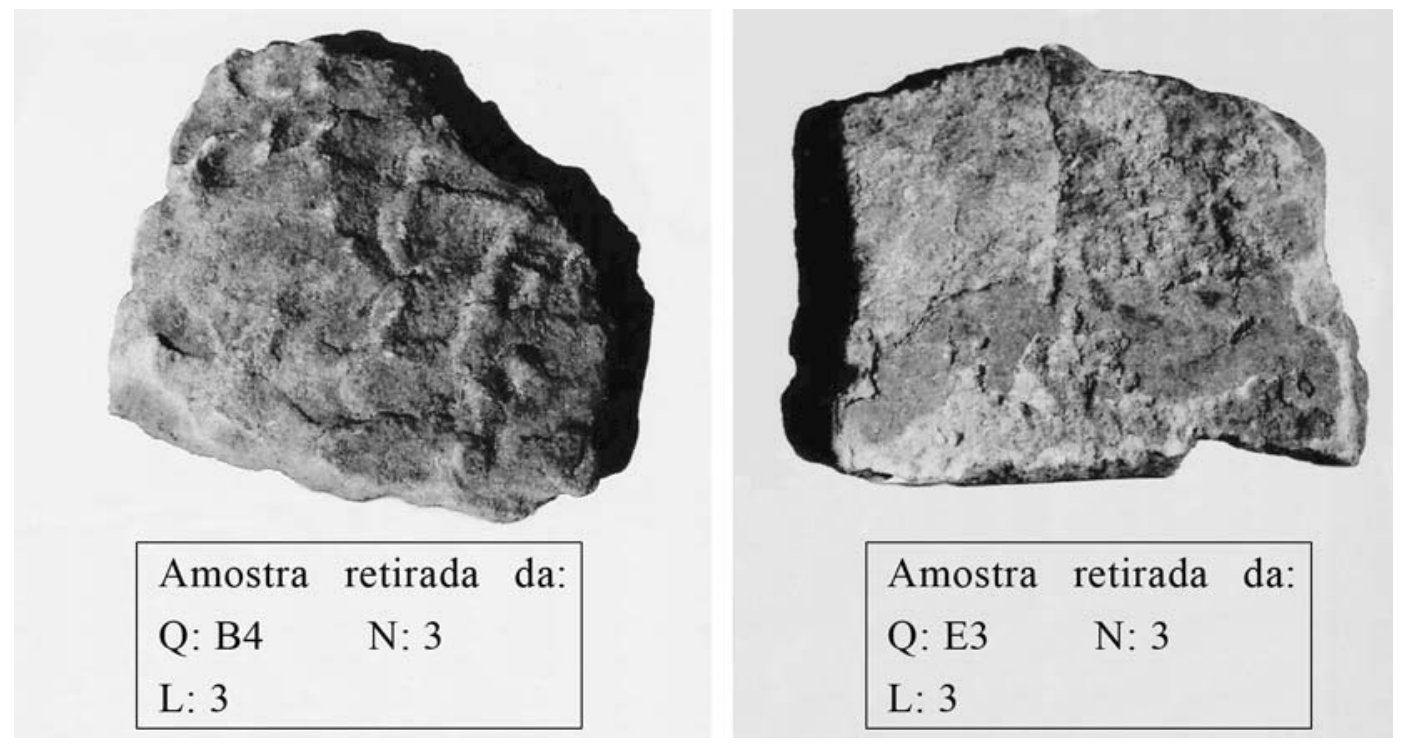

Fig. 2 - Cerâmicas de Tubarão-SC.

0,180 mm de diâmetro. Em seguida, foram pesados cerca de $700 \mathrm{mg}$ da amostra, primeiramente lixiviados em uma solução de $5 \mathrm{ml}$ de $\mathrm{HCl}$ $37,7 \%_{(\mathrm{v} / \mathrm{v})}$, mantendo-se em repouso por 40 min. Após, descartou-se o sobrenadante e se enxaguou o resíduo com água destilada. Em seguida, fez-se nova lixiviação, porém desta vez, utilizando-se $5 \mathrm{ml}$ de $\mathrm{HF} 10 \%_{(\mathrm{v} / \mathrm{v})}$. O resíduo ficou em repouso por cerca de 24 horas para secar completamente, sendo então irradiado em um aparelho tipo Siemens Gammatron com taxa de 43cGy/min, instalado no InRad HCSP (Instituto de Radiologia do Hospital das Clínicas de São Paulo).

Realizaram-se, então, em ambiente escuro, medidas de termoluminescência em uma leitora TL modelo Daybreak 110 Automatic System, com uma taxa de aquecimento de $10^{\circ} \mathrm{C} / \mathrm{s}$, com a finalidade de obter a curva de intensidade $\mathrm{TL}$ versus dose em Gy, com o objetivo de se conhecer a dose acumulada. Cabe salientar que cada ponto das curvas de emissão aqui apresentadas é uma média de 5 a 6 medidas. As amostras, com grãos de quartzo com diâmetro inferior a $0,800 \mathrm{~mm}$, foram analisadas por espectometria de massas com plasma (ICP-MS - Elan 6100), para a determinação dos teores de tório e urânio e posterior obtenção da taxa de dose anual.

\section{Resultados e discussões}

As Figuras 3 e 4 representam as curvas de emissão de amostras da cerâmica CE-PP, a primeira obtida na FATEC e a segunda no LACIFID; as Figuras 5 e 6 representam as curvas de emissão de amostras da cerâmica QB4N3L3, a primeira obtida na FATEC e a segunda no LACIFID, e as figuras 7 e 8 representam as curvas de emissão de amostras da cerâmica QB3N3L3, obtidas na FATEC e LACIFID respectivamente. Na Figura 3 as curvas de emissão referem-se à amostra natural, isto é como recebida, e amostras irradiadas com doses gama de 2, 3 e 4 Gy. Na Figura 4, as curvas apresentadas são relativas à amostra natural, e com dosesgama adicionais de 2, 4, 6, 8, 10 e 12 Gy. No LACIFID, as determinações de TL para obtenção das curvas de emissão foram executadas logo após a irradiação, de modo que os picos em baixas temperaturas aparecem altos, sem terem sofrido decaimento em temperatura ambiente.

O importante, em ambos os casos, é a presença do pico, em aproximadamente $345^{\circ} \mathrm{C}$ que, como anteriormente mencionado, não sofre decaimento, provavelmente por milhões de anos, à temperatura ambiente. Esse pico, como demonstrado nas Figuras 3 e 4, cresce com a dose. 


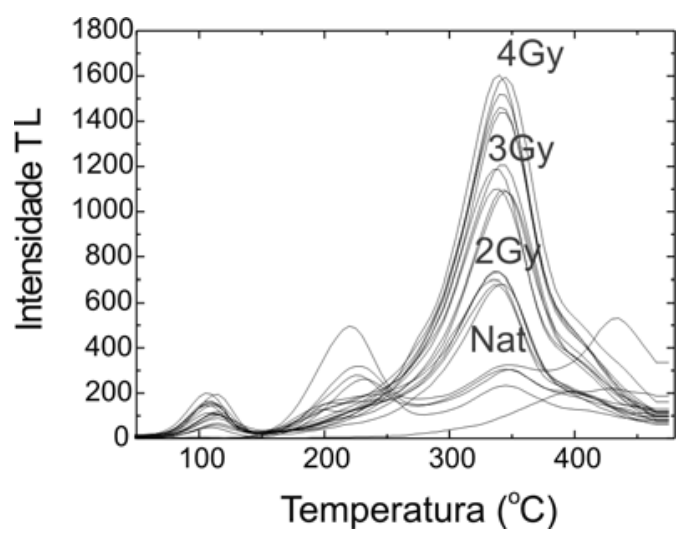

Fig. 3 - Curvas de emissão termoluminescente de grãos de quartzo de CE-PP, obtidas na FATEC.

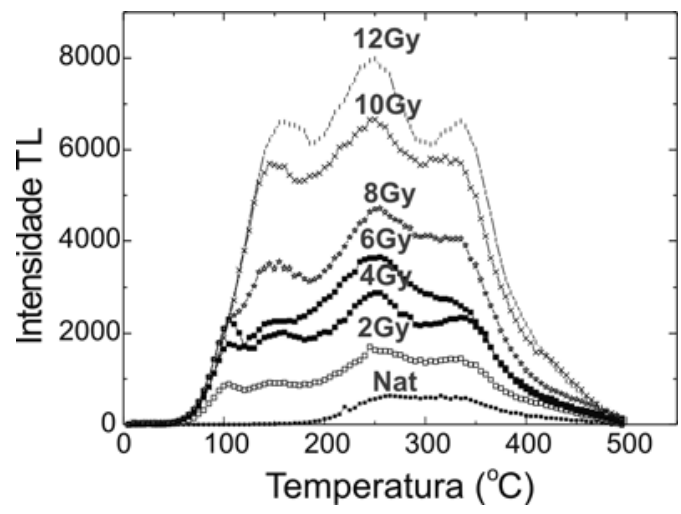

Fig. 4 - Curvas de emissão termoluminescente das mesmas amostras da Figura 3, porém, registradas no LACIFID. A diferença no aspecto da curva de emissão a baixas temperaturas deve-se ao fato de que a curva foi obtida imediatamente após a irradiação.

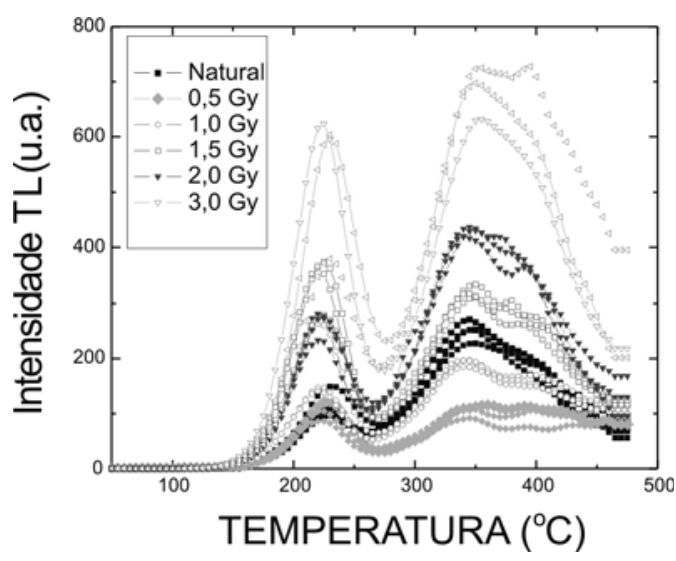

Fig. 5 - Curvas de emissão termoluminescente de grãos de quartzo da amostra QB4N3L3 da UNISUL, obtidas pela FATEC.

\section{Santa Catarina - Amostra QB4N3L3}

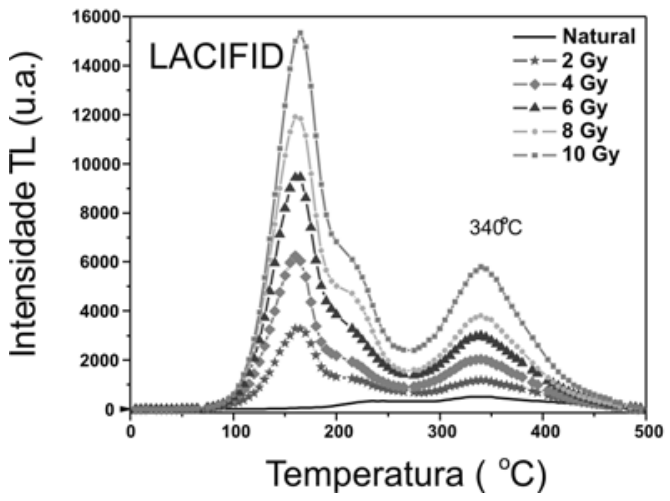

Fig. 6 - Curvas de emissão termoluminescente das mesmas amostras da Fig. 5, obtidas pelo LACIFID.

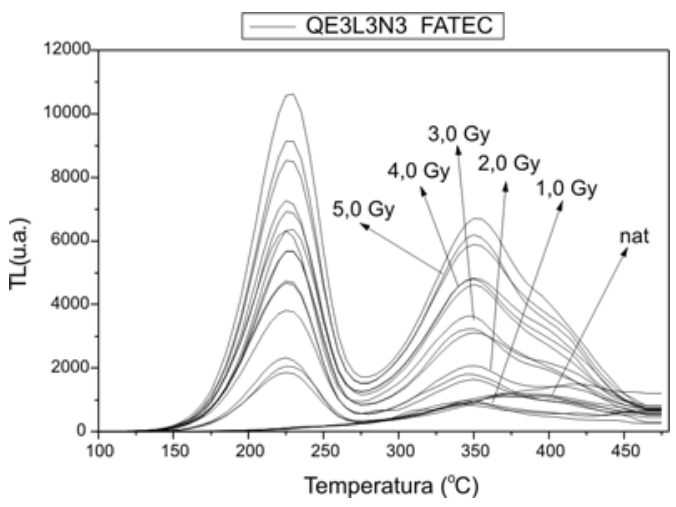

Fig. 7 - Curvas de emissão termoluminescente de amostra QB3N3L3 da UNISUL, obtidas na FATEC.

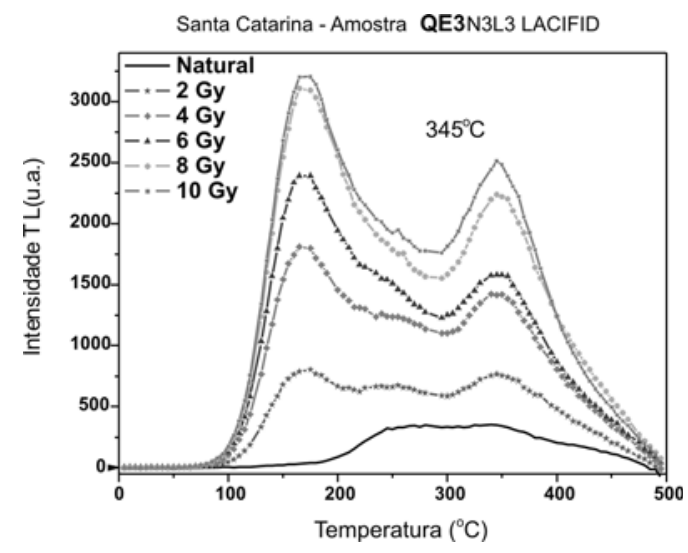

Fig. 8 - Curvas de emissão de termoluminescente emissão da amostra QE3N3L3 obtidas no LACIFID. 
De posse destas informações, é possível obter-se um gráfico da altura do pico de $345^{\circ} \mathrm{C}$ em função da dose. Este gráfico pode apresentar uma correlação linear, para doses baixas e, para doses altas, uma curva exponencial alterando gradualmente para correlação linear (em casos extremos também pode ocorrer a saturação).

No presente caso, a curva foi obtida irradiando-se uma amostra natural com doses adicionais. A extrapolação da reta ou curva ao eixo das doses origina um ponto, cujo valor em Gy fornece a dose acumulada $\left(\mathrm{D}_{\text {ас }}\right)$.

Esse modo para se obter $\mathrm{D}_{\text {ac }}$ é conhecido como método das doses aditivas. Há outros, como o método degenerativo e o de pré-dose, que não serão considerados aqui.

As Figuras 9 e 10 apresentam as curvas de correlação, entre as intensidades e as doses adicionais, usando-se como referência a amostra CE-PP, sendo a primeira do laboratório da FATEC e a segunda do LACIFID. No primeiro caso, obteve-se a $\mathrm{D}_{\text {ac }}=0,84 \pm 0,04 \mathrm{~Gy}$ e, no segundo, $0,91 \pm 0,1 \mathrm{~Gy}$.

As Figuras 11 e 12 apresentam as curvas da intensidade da luz TL do pico de $345^{\circ} \mathrm{C}$ de QB4N3L3 em função da dose, sendo a primeira medida na FATEC e a segunda no LACIFID. Foram obtidas doses acumuladas de 1,24 $\pm 0,08$ Gy e 1,24 $\pm 0,12 \mathrm{~Gy}$, respectivamente.

As Figuras 13 e 14 apresentam as curvas de $\mathrm{I}_{\mathrm{TL}}$ vs dose da amostra QE3N3L3 da UNISUL. A primeira, resultado da FATEC com $\mathrm{D}_{\mathrm{ac}}=1,11 \pm 0,06 \mathrm{~Gy}$ e, a segunda, do LACIFID com $D_{\text {ac }}=1,25 \pm 0,12 \mathrm{~Gy}$.

Cada curva de emissão é obtida fazendo-se 5 a 6 medidas, assim como as retas da Figuras 9, 10, 11, 12, 13 e 14. Apesar disso, não é incomum (e usualmente acontece em medidas experimentais) os pontos se distribuírem com flutuação, de modo a ser possível um ajuste alternativo dos pontos experimentais. Na Figura 14 tem-se, assim, uma segunda reta plausível de ajuste, dando valor distinto de $\mathrm{D}_{\mathrm{ac}}$. São apresentados dois ajustes para evidenciar que, não existe um ajuste “absoluto". Observa-se, no entanto, que quanto maior o número de pontos experimentais, maior a semelhança com o ajuste "absoluto", desde que todos os pontos caiam em uma mesma curva retilínea.

Certamente, isto é uma possível fonte de resultados diferentes obtidos em dois laboratórios ou, como neste caso, no mesmo laboratório.

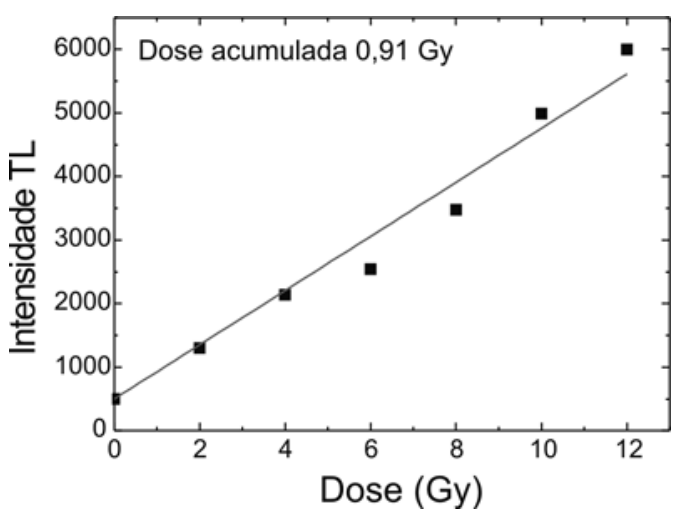

Fig. 9 - Intensidade TL do pico de $345^{\circ} \mathrm{C}$ da cerâmica CE-PP, em função da dose, medidas feitas na FATEC, com o resultado de $D_{a c}$ de aproximadamente $(0,84 \pm 0,04) \mathrm{Gy}$, com $D_{\text {an }}(1,92 \pm 0,17) \mathrm{mGy} /$

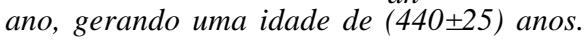

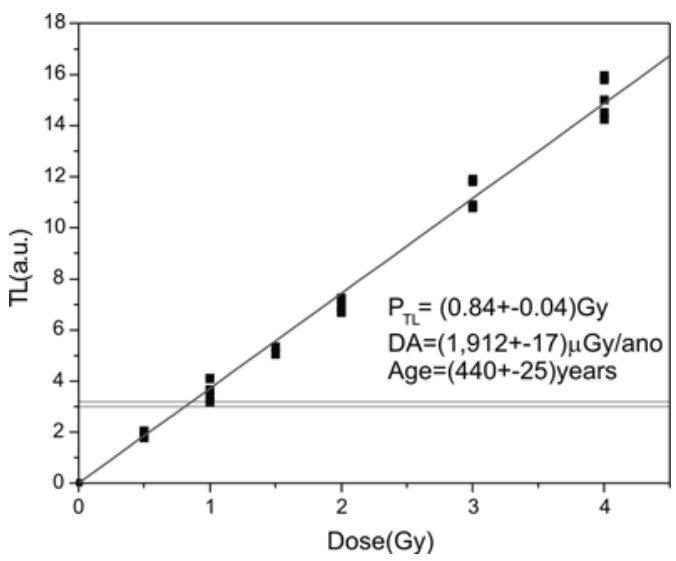

Fig. 10 - Intensidade $T L$ do pico de $345^{\circ} \mathrm{C}$ de CE$P P$ em função da dose. Medidas do LACIFID. $D_{a c}$

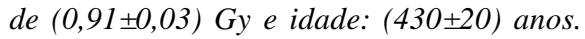

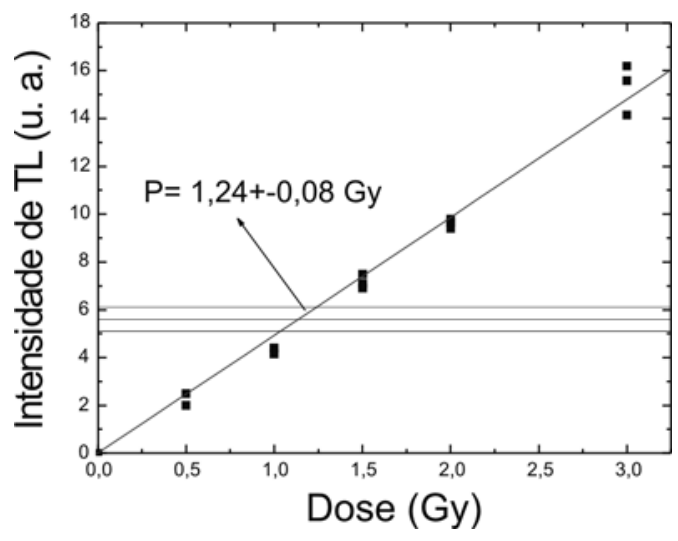

Fig. 11 - Intensidade $\mathrm{TL}$ do pico de $345^{\circ} \mathrm{C}$ da amostra QB4N3L3 da UNISUL, em função da dose. A medida foi feita na FATEC, obteve-se $D_{a c}=(1,24 \pm 0,08) G y$. 


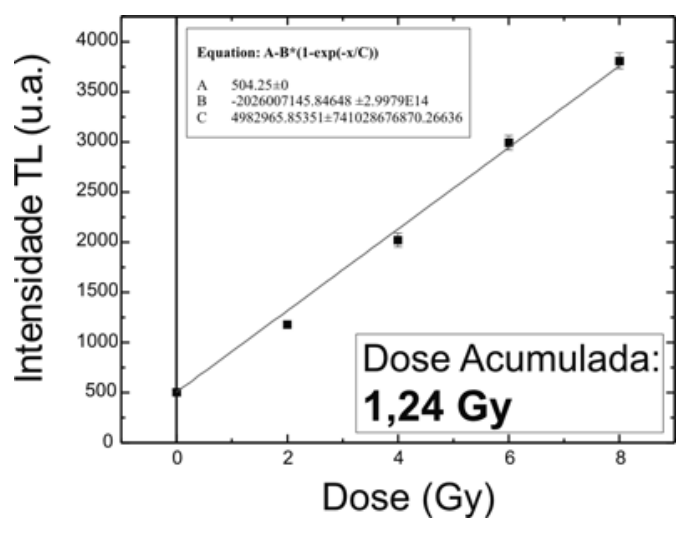

Fig. 12 - Intensidade $T L$ do pico de $345^{\circ} \mathrm{C}$ da amostra QB4N3L3 da UNISUL, em função da dose. A medida foi feita no LACIFID, com $D_{a c}=(1,24 \pm 0,12) G y$.

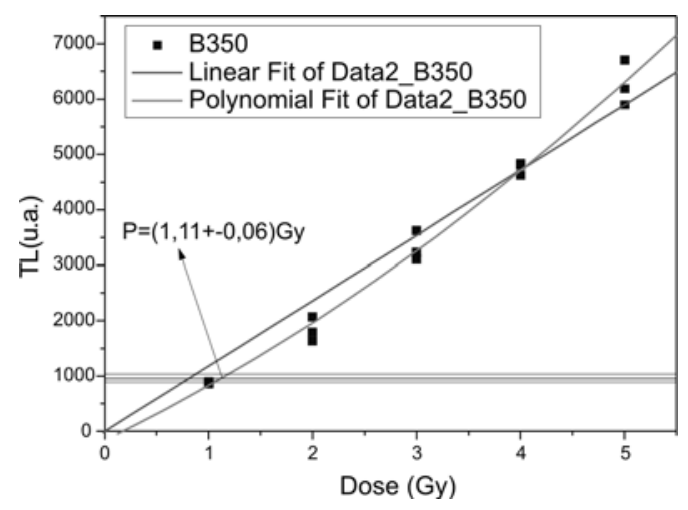

Fig. 13 - Intensidade $T L$ do pico de $345^{\circ} \mathrm{C}$ da amostra QE3N3L3 da UNISUL, em função da dose, medida na FATEC. $D_{a c}=(1,11 \pm 0,06) G y$.

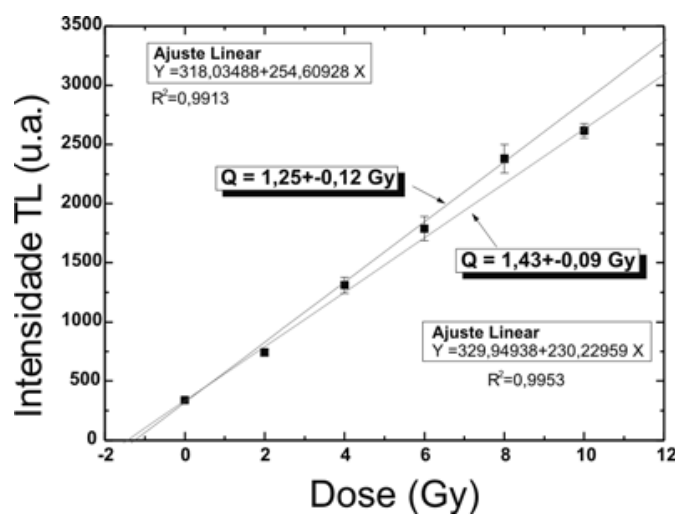

Fig. 14 - Intensidade TL do pico de $345^{\circ} \mathrm{C}$ da amostra QE3N3L3 da UNISUL, em função da dose, medida feita no LACIFID. $D_{a c}=(1,25 \pm 0,12) \mathrm{Gy}$. Um segundo ajuste possível fornece $D_{a c}=(1,43 \pm 0,09) G y$.
Na OSL- luminescência oticamente estimulada, como o nome diz, o cristal que foi irradiado pela radiação natural ou no laboratório, pode emitir luz quando é irradiado com a luz UV ou laser de IV. Na TL, é o calor que provoca a emissão da luz.

A Figura 15 mostra a reta de $\mathrm{I}_{\text {osl }}$ vs. Dose da cerâmica CE-PP. Foi obtida $\mathrm{D}_{\text {ac }}$ de aproximadamente de 0,91 Gy.

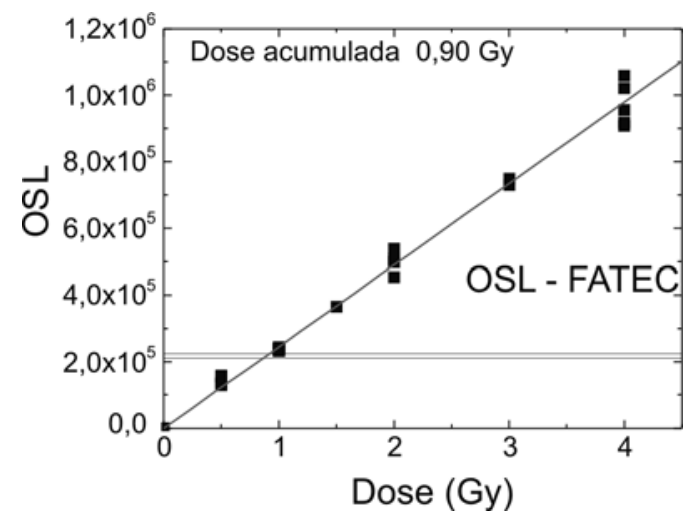

Fig. 15 - Medida de OSL de $D_{a c}$, efetuada na FATEC. $D_{a c}=0,90$ Gy para CE-PP.

As Tabelas II e III apresentam os resultados obtidos em dois laboratórios para dose acumulada $\mathrm{D}_{\mathrm{ac}}$.

TABELA 2

\begin{tabular}{ccc}
\hline \multicolumn{2}{l}{ Dose acumulada $\mathbf{D}_{\text {ac }}$ (em Gy), na cerâmica CE-PP } \\
\hline \hline & FATEC & LACIFID \\
\hline Por TL & $0,84 \pm 0,04$ & $0,91 \pm 0,03$ \\
Por OSL & $0,90 \pm 0,05$ & - \\
\hline
\end{tabular}

TABELA 3

\begin{tabular}{ccc}
\hline $\begin{array}{c}\text { Dose acumulada D } \\
\text { QB4N3L3 e QE3N3L3 }\end{array}$ \\
\hline \hline & FATEC & LACIFID \\
\hline Por TL na QB4N3L3 & $1,24 \pm 0,08$ & $1,24 \pm 0,12$ \\
Por TL na & $1,11 \pm 0,06$ & $1,25 \pm 0,12$ \\
QE3N3L3 & & $1,43 \pm 0,09$ \\
\hline
\end{tabular}




\section{Conclusões}

1) As datações foram efetuadas em uma única cerâmica de Presidente PrudenteSP (UNESP), e em duas cerâmicas de Tubarão-SC (UNISUL). Os resultados obtidos para dose acumulada, no laboratório da FATEC e no laboratório LACIFID, apresentaram boa coerência dentro do erro experimental.

2) Contudo, é bom observar que dados confiáveis dependem de vários fatores como:

- Informar, com clareza, as condições da coleta das amostras, como, por exemplo: profundidade da coleta, umidade de solo, coletar, além da peça de cerâmica, uma amostra do solo onde a cerâmica estava, etc. Detalhando-se portanto, todas as condições ambientais, isto é, se o local de coleta é um pântano, se a amostra veio de uma camada de pequena profundidade, se a superfície foi submetida a queimadas, se a amostra foi exposta à radiação solar intensa etc..

- Aparelho de leitura em bom estado de funcionamento e bem calibrado. Sugere-se que inter-calibrações periódicas sejam realizadas entre os laboratórios que atuam na área.

- Medidas realizadas por pessoal devidamente treinado, o ideal é que sejam feitas por estudantes de física, ou técnicos com sólidos conhecimentos em física.

\section{Agradecimento}

Agrademos à Dra Laura Furnari do InRad HC - FMUSP pela irradiação das amostras O presente trabalho foi financiado parcialmente pela FAPESP e pelo CNPq.

WATANABE, S.; TATUMI, S.H.; FARIAS, T.M.B.; SOUZA, S.O.; GENNARI, R.F.; KUNZL, R.; FARIAS, D.S.E. Confiability test of thermoluminesce (TL) and optically stimulated luminescence (OSL) methods. Rev. do Museu de Arqueologia e Etnologia, São Paulo, 15: 383-391, 2005.

ABSTRACT: The knowledge of the age of a sample is of great importance for researchers in archeology and geology, among other sciences. There are several dating methods for archaeological and geological materials. To begin with there is the well known radiocarbon and radioactivity methods,based on the decay of the C-14 isotope or other isotopes with appropriate half-life. Of increasing importance one finds those based on the energy deposition in solids. Thermoluminescence (TL) or optically stimulated and electron paramagnetic resonance (EPR) are solid state process dependent on energy deposited by incident ionizing radiation in solids.

Some local archaeologists questioned the trustfulness of TL and OSL methods for dating potteries.

In the present work three large potsherds, each one divided into two parts were dated at two of the most active dating laboratory to crosscheck their dating results. A reasonably good agreement between the results was obtained indicating that the methods when correctly applied are trustable.

UNITERMS: Thermoluminesce-Dating-OSL-Ceramics. 
WATANABE, S.; TATUMI, S.H.; FARIAS, T.M.B.; SOUZA, S.O.; GENNARI, R.F.; KUNZL, R.; FARIAS, D.S.E. Testes de confiabilidade dos métodos de datação por termoluminescência (TL) e luminescência oticamente estimulada (OSL). Rev. do Museu de Arqueologia e Etnologia, São Paulo, 15: 383-391, 2005.

\section{Referências bibliográficas}

\author{
AITKEN, M.J. \\ 1985 Thermoluminescence dating. London: \\ Academic Press. \\ CAMERON, J.R.; SURTHARALINGAM, N.; KENNY,A.R. \\ 1968 Thermoluminscence dosimetry. Madison: \\ Univ. Wisconsin Press. \\ CHEN, R.; MC KEEVER, S.W.S. \\ 1997 Theory of Thermoluminescence and related \\ Phenomena. Singapore: World Scientific. \\ IKEYA, $M$. \\ 1993 New application of electron spin resonance. \\ Singapure: World Scientific. \\ KRBETSCHEK, M.R.; GOTZE J.; DIETRICH, A.; \\ TRAUTMANN T. \\ 1997 Spectral Information from minerals relevant to \\ luminescence dating. Radiation Measurements, \\ 27: 695-748. \\ MC KEEVER, S.W.S. \\ 1988 Thermoluminescence of solids. Cambridge: \\ Cambridge Univ. Press.
}

Recebido para publicação em 7 de março de 2006.
ROQUE, C.; GUIBERT, P.; VARTANIAN, E.; VIEILLEVIGNE, E.; BECHTEL, F.

2004 Changes in luminescence properties induced by thermal treatments; a case study at Sipan and Trujillo Moche sites (Peru). Radiation Measurements, 38 (1): 119-126.

SASTRY, M.D.; SULLASI, H.S.L.; CAMARGO, F.; WATANABE. S.; PROUS, A.P.P.; SILVA, M.M.C.

2004 Dating sediment deposits on Montalvanian carvings using EPR and TL methods. Nuclear Instruments \& Methods In Physics Research Section B-Beam Interactions With Materials And Atoms, 213: 751-755.

TATUMI, S.H.; MARTINS, G.R.; KASHIMOTO, E.M.; AYTA, W.E.F.; WATANABE, S.

1998 Thermoluminescence dating of archaeological ceramics collected from State of Mato Grosso do Sul, Brazil. Radiation Effects And Defects In Solids, 146 (1-4): 297-302. 